\title{
Article
}

\section{Developing and Assessing Curriculum on the Physics of Medical Instruments}

\author{
Warren Christensen, ${ }^{*}$ James K. Johnson, ${ }^{\dagger}$ Grace R. Van Ness, ${ }^{\dagger}$ Elliot Mylott, ${ }^{\dagger}$ \\ Justin C. Dunlap, ${ }^{+}$Elizabeth A. Anderson, ${ }^{+}$and Ralf Widenhorn ${ }^{\dagger}$
}

${ }^{*}$ Department of Physics and School of Education, North Dakota State University, Fargo, ND 58108; ${ }^{\dagger}$ Department of Physics, Portland State University, Portland, OR 97207

Submitted September 2, 2012; Revised February 28, 2013; Accepted March 5, 2013

Monitoring Editor: Eric Brewe

\begin{abstract}
Undergraduate educational settings often struggle to provide students with authentic biologically or medically relevant situations and problems that simultaneously improve their understanding of physics. Through exercises and laboratory activities developed in an elective Physics in Biomedicine course for upper-level biology or pre-health majors at Portland State University, we aim to teach fundamental physical concepts, such as light absorption and emission and atomic energy levels, through analysis of biological systems and medical devices. The activities address the properties of electromagnetic waves as they relate to the interaction with biological tissue and make links between physics and biomedical applications such as microscopy or laser eye surgery. We report on the effect that engaging students in tasks with actual medical equipment has had on their conceptual understanding of light and spectroscopy. These initial assessments indicate that students' understanding improves in some areas as a result of taking the course, but gains are not uniform and are relatively low for other topics. We also find a promising "nonshift" in student attitudes toward learning science as a result of taking the course. A long-term goal of this work is to develop these materials to the extent that they can eventually be imported into an introductory curriculum for life sciences majors.
\end{abstract}

DOI: $10.1187 /$ cbe.12-09-0142

Address correspondence to: Warren Christensen (warren .christensen@ndsu.edu).

E.M., J.C.D., and R.W. were involved with the curriculum development, which involved planning the course, designing the activities, instructing the course, creating learning goals, creating questions, and choosing assessment questions. W.C., J.K.J., G.V.N., E.A.A., and R.W. were involved with the assessments, which involved creating and choosing questions on the OAPA, creating learning goals, creating quiz questions, preparing the CLASS survey and interpreting the results. R.W. was the lead developer for the curriculum and instructor for both the Physics in Biomedicine course and the algebra-based physics course. W.C. was the lead assessor, choosing the format of questions for the quizzes and played the largest role in interpreting the data.

(C) 2013 W. Christensen et al. CBE-Life Sciences Education (C) 2013 The American Society for Cell Biology. This article is distributed by The American Society for Cell Biology under license from the author(s). It is available to the public under an AttributionNoncommercial-Share Alike 3.0 Unported Creative Commons License (http:/ / creativecommons.org/licenses/by-nc-sa/3.0).

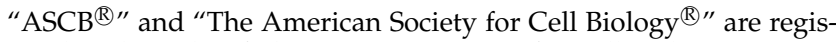
tered trademarks of The American Society for Cell Biology.

\section{INTRODUCTION}

The majority of investigations and research-based instructional reforms from physics education research (PER) have been aimed at the introductory calculus-based level. On the whole, research into student thinking and learning in algebrabased physics courses, which are populated primarily by biology and life sciences majors, has been sorely lacking. The work that has been done includes holistic course changes and seems effective on a number of measures (Hammer and Redish, 2009; Meredith and Bolker, 2012). Many of the pedagogical reforms that have been proposed by textbook authors are "biological application" problems that are largely superficial rather than biologically authentic and/or relevant to majors within the biological sciences (Watkins et al., 2012).

This paper provides insights into initial attempts at assessing instructional materials and laboratory activities developed to teach core physics principles in a biologically relevant manner. These materials are used within an upper-level course called Physics in Biomedicine. The course focuses on biomedical equipment and the physics involved in their use. For example, lasers for surgery, magnetic resonance imaging (MRI), and computed tomography (CT) rely on electromagnetic waves and their interaction with different tissues in the 
body. Optics and atomic and nuclear physics have significant relevance, and the course attempts to increase students' understanding of these topics, while increasing their interest in both physics and biology.

The authors understand that most institutions might not be able to add a similar course to what is often a full docket of classes. However, as institutions search for authentic experiences for students that cross the boundary of physics and biology for the introductory sequence, we feel that some activities will be importable into the introductory algebra-based physics sequence at a future date.

Throughout the course, we monitored students' conceptual understanding and their attitudes. We used a combination of free-response and multiple-choice items before, during, and after the course of instruction in the class. These initial assessments indicate that students' understanding improves overall as a result of taking the course, but gains for certain topics are relatively low. We also find a nonshift in student attitudes toward learning science as a result of taking the course, which is generally viewed as a positive outcome (Adams et al., 2006).

\section{Background for Course Development}

Physics in Biomedicine, an intermediate-level physics course developed at Portland State University (PSU), was first offered in the Summer of 2008. It is an intensive 3-wk summer course that meets daily, with 14 class periods of 140 min each. This paper describes the results from the course offered during Summer 2012.

The course structure included four pedagogical strategies: lectures by the physics professor (R.W.), guest lectures by biomedical researchers or physicians, classroom discussions, and laboratory activities. Laboratory work included both virtual activities and traditional hands-on laboratory exercises. The course is centered on how different types of electromagnetic waves are generated, how they interact with tissue, and how this is utilized in the instruments used by researchers and physicians.

This theme is extended to include ultrasound imaging and certain aspects of MRI. The ultrasound imaging segment of the course allowed discussion of the similarities and differences of electromagnetic waves and sound waves. MRI uses electromagnetic waves to stimulate spin flips in the hydrogen atom and also utilizes static magnetic fields. Contrasting the generation of a static magnetic field with electromagnetic waves tied MRI well into other course topics. The structure of the course covered topics of increasing complexity as the class progressed. Due to the high complexity of the subject of MRI, it was taught over three class periods at the end of the course.

The course assumes that students know the material typically taught in introductory general physics (mechanics, thermodynamics, electromagnetism, and optics) and transitions after a review of optics to advanced topics in atomic and nuclear physics. While most introductory physics textbooks contain chapters on atomic and nuclear physics, these topics are typically underrepresented in introductory courses. Additionally, because the course is at an introductory level with a variety of majors enrolled, a focus on medical applications is not typical (Redish and Hammer, 2009).

The course described here covers biomedical concepts in the following sequence. It starts off with biomedical applica- tions of optics: laparoscopy, laser eye surgery, pulse oximetry, and optical microscopy. After the optics portion, an overview of radiological techniques was given, followed by lectures and activities on ultrasound imaging, CT scans, and positron emission tomography. Before the MRI section, the use of radiation to treat cancer was discussed.

The guest lectures were typically PowerPoint presentations incorporating the use of physics principles in the lecturer's field of expertise. The presenters included radiologists, oncologists, radiation physicists, ophthalmologists, microscopists, or biologists. Lectures by R.W. on the physics background were done either in PowerPoint or with the use of a blackboard.

While it is beyond the scope of this article to describe each part of the course, the following illustrates a typical sequence of activities. In conjunction with a radiologist's lecture on imaging modalities in medicine, the students conducted a laboratory exercise on CT that included both a computer simulation and a hands-on component (Mylott et al., 2011). After the laboratory exercise, students were assigned a reading on the use of CT in clinical practice. Following that, a class discussion facilitated students in weighing the benefits of early detection and diagnosis versus the risk associated with radiation (Berenson and Abelson, 2008). Students were encouraged to integrate ideas from the preceding activity, instruction, and reading.

The ophthalmologists' lecture on LASIK surgery was coupled with computer exercises on lasers and a worksheet on light absorption, which led to a laboratory exercise on pulse oximetry. The activity on lasers was given as a worksheet for a Java-based Physics Education Technology (PhET) simulation that students worked on in groups of two or three using computers in the physics teaching laboratory (Perkins et al., 2006; University of Colorado, 2011). After completion of that lab, students were assigned a worksheet on light absorption as an independent homework activity. In it, students interpreted a graph displaying the absorption spectrum of hemoglobin, oxygenated hemoglobin, water, and melanin. Students found absorption coefficients for different wavelengths from a logarithmic graph, calculated penetration depths, and related the results to laser surgery.

The light absorption worksheet is also relevant to the pulse oximetry device. It takes advantage of different absorption characteristics of hemoglobin and oxygenated hemoglobin. This was explored in another laboratory exercise in which students used bromothymol blue as a blood analogue and measured its absorption spectrum using a low-cost spectrograph (Vernier, www.vernier.com/products/sensors/ spectrometers/svis-pl). Bromothymol blue changes color in response to varying levels of $\mathrm{CO}_{2}$ content; a change in $\mathrm{CO}_{2}$ level can be easily achieved by exhaling through a straw into the solution. Students observe how this changes the absorption spectrum and explore in the next steps how two differentcolored light-emitting diodes can be used to extract information on the $\mathrm{CO}_{2}$ content in a manner similar to the medical pulse oximetry device obtaining information on the oxygen content of blood.

The course, in general, flows well, is enjoyable to teach, and, based on evaluation surveys, is overwhelmingly enjoyed by students in comparison with the algebra-based physics course. Students typically seem more engaged than during the introductory physics sequence. 


\section{Demographics}

Physics in Biomedicine has a unique enrollment that requires special attention. This course is most commonly taken by upper-division students who have previously taken introductory physics courses. The course is not required for any major, so these students are self-selecting for this course out of interest and to fulfill an elective physics or biology course. In Summer 2012, 24 students were enrolled in the course, with 23 students consenting to participate in this study (Table 1). Of the 23 participants, 14 were male and 9 were female. This is in slight contrast to past years, when female students slightly outnumbered male students. These students were primarily upperclassmen $(56.5 \%)$ and postbaccalaureate students $(26.1 \%)$. The largest group of students was biology majors $(43.5 \%)$; other majors included general science $(21.7 \%)$ and computer science, engineering, and physics (21.7\%). One enrolled student was majoring in anthropology. The general science track is an interdisciplinary major at PSU, which allows for both concentration and diversification of course work. Health science preprofessional students preparing for medical, dental, or other health careers at PSU find it efficient to coordinate their professional school prerequisites into the general science major and often enroll into this track.

Of those enrolled in the course, the majority of students were interested in attending medical school $(65.2 \%)$, while a smaller proportion of students wanted to attend graduate school. One student aspired to enroll into an $\mathrm{MD} / \mathrm{PhD}$ program.

Surveys prior to enrollment into the Physics in Biomedicine course revealed a large number of students completed the majority of science requirements for medical school: general biology $(91.3 \%)$, general chemistry $(100 \%)$, and general physics $(91.3 \%)$. Approximately half of the students completed courses in anatomy and physiology after their general biology course.

Although all of the students completed general chemistry, a smaller proportion of those students had completed organic chemistry (60.9\%). Further, only one student completed advanced physics and chemistry courses.

Medical school requirements do not include mathematics courses. However, good mathematical skills are necessary to succeed in physical science classes. A large proportion of Physics in Biomedicine students completed algebra 1 and 2 courses in their college career (73.9 and 69.8\%, respectively). We found that $61 \%$ of the students had completed calculus $1(30 \%$ of biology majors and all of the general science majors). Smaller proportions of the Physics in Biomedicine cohort went on to complete calculus 2 (39.1\%), calculus 3 $(39.1 \%)$, and calculus $4(21.7 \%)$. One student completed advanced mathematics courses beyond differential equations.

\section{METHODS}

We used a modified backward design to assess the effectiveness of the instruction (Wiggins and McTighe, 2005). We identified desired learning outcomes that were targeted within the Physics in Biomedicine course, and we developed questions to assess those outcomes. The project began with the idea of tying biomedical equipment to physics principles within the classroom. Laboratory activities and worksheets were created
Table 1. Physics in Biomedicine 2012 student demographic data

\begin{tabular}{ll}
\multicolumn{1}{c}{ Characteristic } & Number $(\%)$ \\
\hline Year in college & \\
Freshman & 0 \\
Sophomore & 0 \\
Junior & $3(13)$ \\
Senior & $13(56.5)$ \\
Postbaccalaureate & $6(26.1)$ \\
Other & $1(4.3)$ \\
Major & $1(4.3)$ \\
Anthropology & $10(43.5)$ \\
Biology & $3(13)$ \\
Computer science/engineering & $5(21.7)$ \\
General science & $2(8.7)$ \\
Health science & $2(8.7)$ \\
Physics & \\
Career aspirations & \\
Graduate school & $4(17.4)$ \\
Medical school & $15(65.2)$ \\
MD/PhD program & $1(4.3)$ \\
Neither graduate nor medical school & $3(13.0)$ \\
Total & $23(100)$ \\
\hline
\end{tabular}

${ }^{\text {a }}$ Responses not mutually exclusive. $N=23$.

by the course instructor (R.W.) and physics graduate students (J.C.D. and E.M.) and used in teaching the course during a summer session several times before a coordinated effort to investigate the materials' pedagogical effectiveness began. A traditional backward-design model would have used the assessments to inform the creation and design of the curriculum, but a good deal of our curriculum was prepared before significant attempts at assessment began. However, in keeping with backward design, we will modify the existing activities based on the results of our preliminary work.

Learning goals were identified for the course as a whole and for individual modules.

\section{Content Assessments}

The research team determined a set of content goals for the course. We also identified a number of concepts that the instructor was expecting students to understand coming into the course. The learning goals revolve around concepts of atomic and nuclear physics, as well as optics and its relation to human tissue.

A search of the PER literature reveals few studies on student understanding of these topics. Work by Ambrose et al. (1999) investigates student thinking on relationships between formalism and physical phenomena in the context of electromagnetic waves, while Vokos et al. (2000) looked at students thinking about diffraction and interference of light. Ultimately, the findings of these studies, while seemingly similar to our investigation of electromagnetic waves, do not probe concepts that our curriculum is attempting to teach, and are therefore of limited use for this study. Schlingman et al. (2012) report on their validation study of the Light and Spectroscopy Conceptual Inventory (LSCI), which probes student thinking about concepts of light and spectroscopy within the context of astronomy. Although the majority of questions were not appropriate, as they deal with concepts relating to celestial 
bodies, we were able to use several of the questions from the LSCI with permission of the authors. ${ }^{1}$

We identified learning outcomes for individual modules within the broader course. These outcomes in physics, biology, and mathematics were identified by the instructor as concepts he expected students to know coming into the course or concepts they would learn through instruction and activities. We developed a number of assessments to probe student thinking on those ideas.

These unit-specific assessment questions are a combination of free-response and multiple-choice questions that were given both before and after instruction on a given module.

Questions were developed for five different modules within the course: light and absorption, lasers, pulse oximeter, CT, and MRI. Due to page restraints, we will address only two of the modules in this article: light absorption and pulse oximetry. The time between pre- and postinstruction quizzes differed depending on the learning module, but the pre- and postinstruction testing occurred before and after all instruction on that particular module. ${ }^{2}$

\section{Attitudinal Assessment}

A well-documented finding in PER is that, despite the best efforts of instructors, nearly all physics instruction at the introductory level leads to a shift away from expert-like opinions about science and science learning (e.g., Moore and Foy 1997; Redish et al., 1998; Adams et al., 2006). An example being that experts view science as ever-changing, but students tend to view science as a collection of facts that are to be memorized. There are only a few documented cases of instruction that produce significant positive attitudinal shifts, such as Workshop Physics (Laws et al., 1999) or modeling instruction (Brewe et al., 2009). These curricula share a common feature of students learning physics through doing laboratory exercises that are not "cookbook" in nature. Rather, they require students to test hypotheses that they come up with themselves; students are frequently asked to make predictions and reflect back on their predictions after conducting an experiment. These curricula engage students in the real phenomena for the majority of class time.

Given the focus on laboratory activities and real-world applications featured in the Physics for Biomedicine course, it seemed plausible that it would be a good candidate for improving student attitudes toward physics. Assessing attitudinal shifts at the upper division is rare but not unprecedented (McKagan et al., 2007). We needed the most appropriate assessment tool to measure the attitudes of the students in our course. A number of instruments have been developed to assess aspects of students' attitudes and ideas toward science and science learning, for example, the Maryland Physics Expectations Survey-MPEX (Redish et al., 1998), the Epistemologicial Beliefs Assessment for Physical Science-EBAPS (Elby, 2001), and the Views About Sciences Survey-VASS

\footnotetext{
${ }^{1}$ We are in the process of developing additional questions that probe students' thinking, and future work may include construction of a valid and reliable assessment instrument for optical and atomic physics concepts.

${ }^{2}$ Samples of assessment materials, lab exercises, and worksheets can be found at http://web.pdx.edu/ ralfw/instructional-material .html or can be obtained by contacting R.W. (ralfw@pdx.edu).
}

(Halloun, 1996). We used the Colorado Learning Attitudes toward Science Survey (CLASS) to measure the course's effect on attitudes (Adams et al., 2006).

We chose the CLASS survey for two reasons. First, its material is the combination of the most robust portions of the other instruments. Second, it does this with stronger bias controls. When naming and defining categories of student thought, the CLASS developers placed more emphasis on observations of how responses correlate (using exploratory factor analysis) than on traditional notions of how the statements are related to each other.

The CLASS groups its questions into eight categories (see Figure 1). Because these categories are less strongly linked to familiar notions, interpreting the significance of a category is best accomplished by looking at its individual questions. Additionally, CLASS data are reported with two other categories: Overall and All Categories. Some questions do not fit in any of the categories but are valid and useful. Overall includes such freestanding questions, while All Categories does not. The CLASS uses a Likert scale for rating agreement on 42 statements. The scoring standard for the CLASS is the average response of physics experts. It is the most comprehensive instrument currently available to assess attitudes toward physics and physics learning.

The CLASS is used as a pre/postassessment for a term and/or sequence. CLASS data are generally reported as a net shift, positive being toward expert-like (favorable) responses, and negative being toward novice-like (unfavorable) responses. Primarily used in introductory physics courses, it has shown that even research-validated curricula that improve conceptual understanding often produce significant shifts away from expert-like attitudes in students (Brewe $e t$ al., 2009).

\section{RESULTS}

\section{CLASS Data: Demographics and Attitude Shifts}

The population of students in the Physics in Biomedicine course is mostly self-selected from a larger population of students who have previously taken the algebra-based physics courses. Of the 23 students in the study, 21 reported taking a general physics course prior to Physics in Biomedicine. The CLASS was administered as an online survey on the students' own time, after the first and last day of the Physics in Biomedicine course in Summer 2012. It was also given to the algebra-based physics course early and late during Fall 2012 term. Further, a paper copy of the CLASS was given to students on the last day of the Spring 2012 algebrabased physics course (only an exit survey was collected). The CLASS data were scored ordinally (in keeping with the literature) and utilized a scoring system provided by Adams et al. (2006). Responses were scored as "agree," "disagree," or "neutral/conflicted." This third response type means expertlike and novice-like shifts may not be equal and opposite. We report both expert-like and novice-like shifts when reporting on individual questions or presenting absolute scores rather than shifts. The scoring system provided by the CLASS developers reports a shift (in a category) as significant when the shift is more than twice the SE. Specific criteria must be met for data to be included in the analysis. Students can have no 


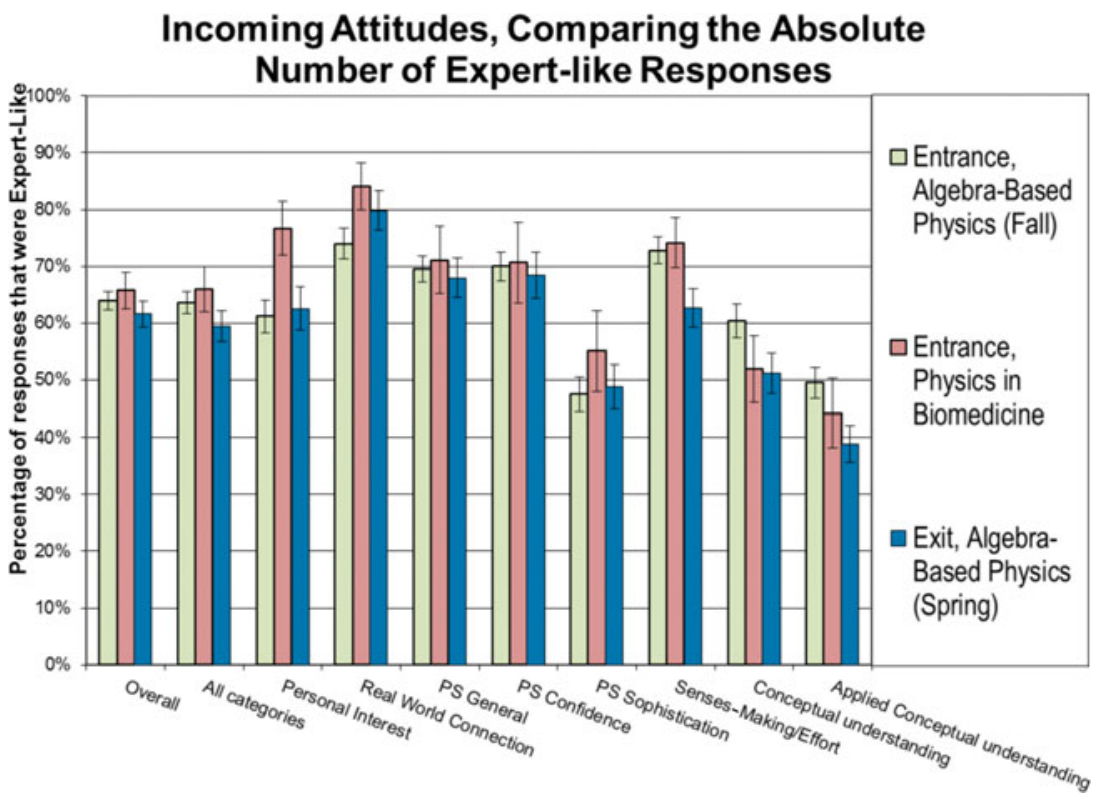

Figure 1. Comparisons of students' expert-like responses to the CLASS survey. Compared are: an exit survey of introductory algebra-based physics in Spring of 2012, an entrance survey of Physics in Biomedicine entrance in Summer of 2012, and an entrance survey of algebra-based physics in Fall of 2012. The categories are Personal Interest, RealWorld Connection, Problem-Solving (PS) General, PS Confidence, PS Sophistication, SenseMaking/Effort, Conceptual Understanding, and Applied Conceptual Understanding. The result for all of the questions within a category is identified as All Categories, and the result for all questions on the survey, even those validated but not included as part of a category, is identified as Overall. Error bars represent SE. more than four missing responses, and only data from students whose exit surveys can be matched to their entrance surveys can be included. All of the participating students in the biomedical course satisfied these criteria $(23 / 23)$, while $47 \%(91 / 192)$ of participating students in the Fall term of algebra-based physics satisfied the criteria.

\section{Demographics According to the CLASS: Self-Selection Effects}

As previously mentioned, Physics in Biomedicine students primarily come from a population of students who have completed algebra-based physics. The CLASS data from Spring term show the final attitudes of students after a full year of introductory physics. By comparing this with the absolute scores of students entering Physics in Biomedicine, we can gain additional insight into the attitudes of students electing to take this course. Additionally, we draw comparisons with students entering the first term of the algebra-based physics sequence in the Fall 2012 term.

We used the average scores of both populations to get an idea of how the Physics in Biomedicine students compare with their peers. Figures 1 and 2 contrast the two populations of algebra-based physics students (those having just finished Spring term and those having just entered Fall term) with the population entering Physics in Biomedicine. In the Overall

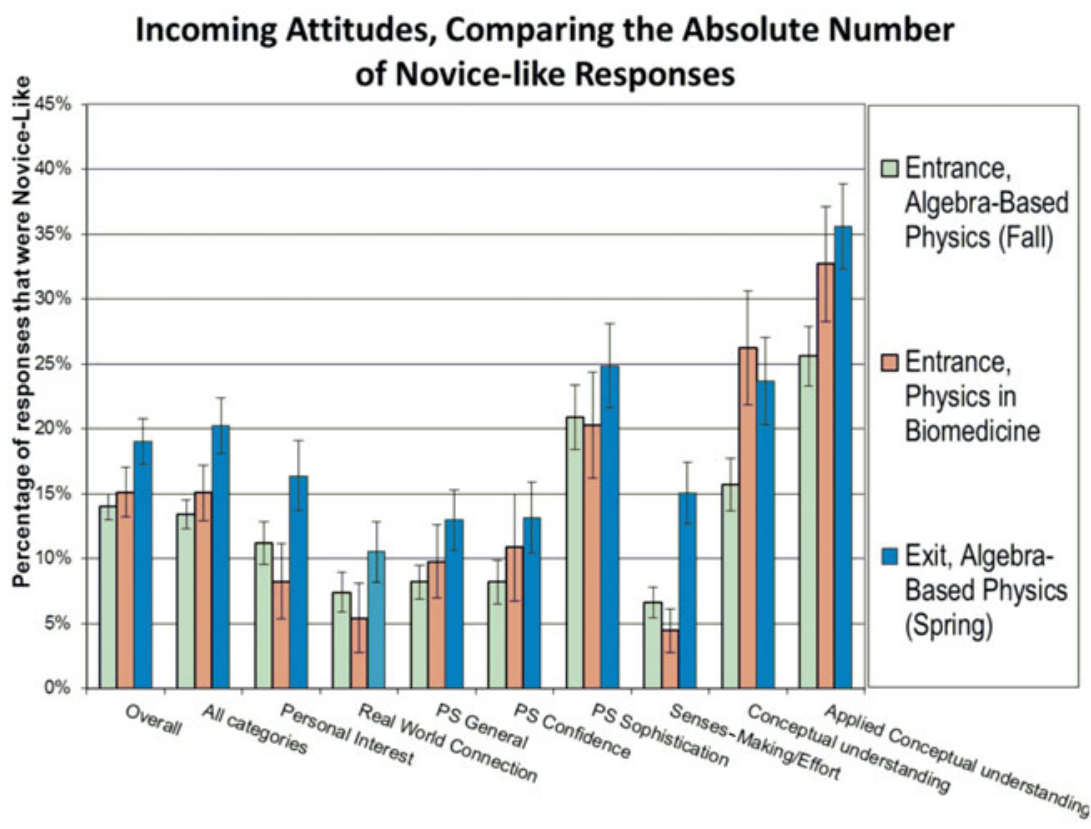

Figure 2. Comparisons of novice-like responses to the CLASS survey. Compared are: an exit survey of introductory algebra-based physics in Spring of 2012, an entrance survey of Physics in Biomedicine entrance in Summer of 2012, and an entrance survey of algebra-based physics in Fall of 2012. Error bars represent SE. See Figure 1 for categories. 
Shifts in Expert-like Responses: Fall Term of Algebra-based Physics and Physics in Biomedicine

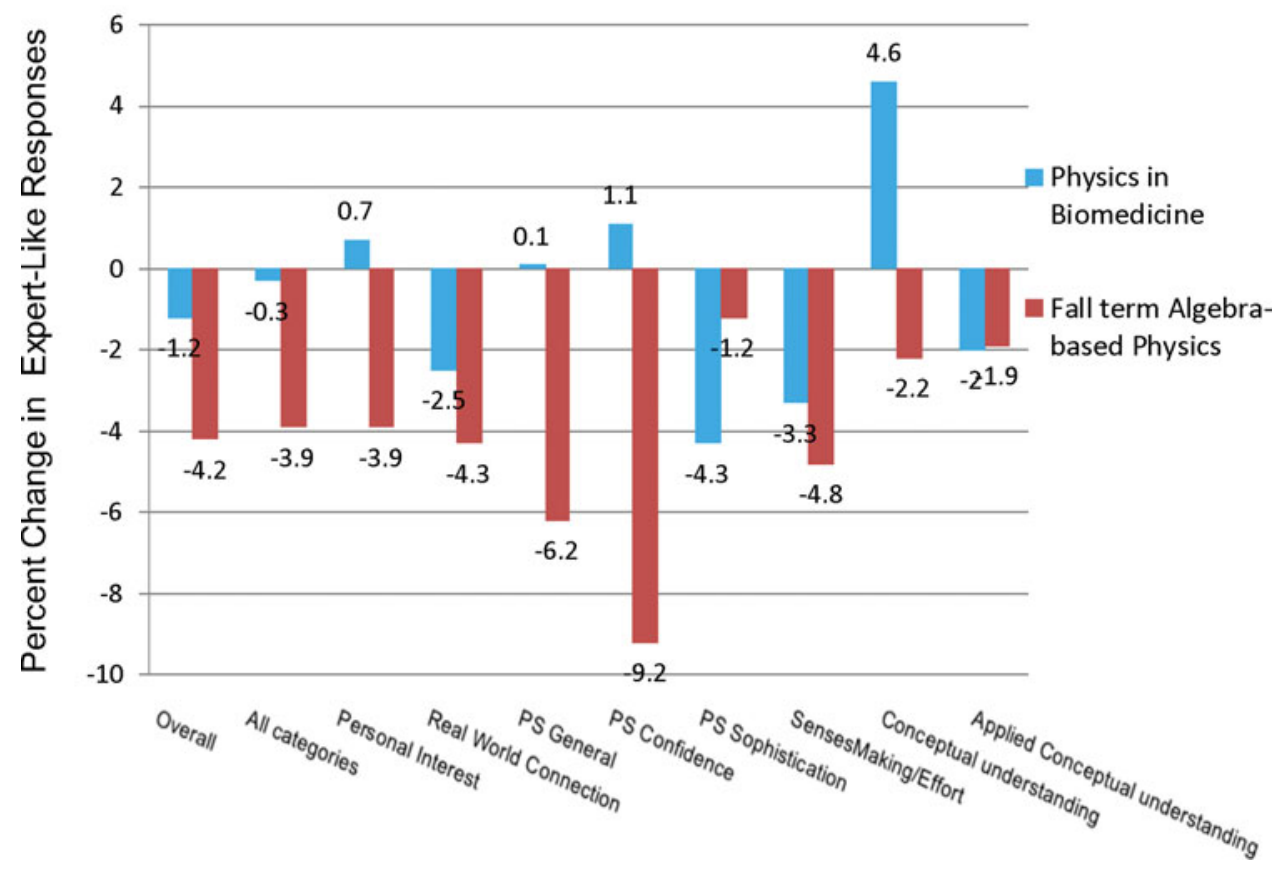

Figure 3. Comparisons of the change in expert-like responses to CLASS survey. Compared are: entrance and exit surveys of introductory algebra-based physics in Fall of 2012 and entrance and exit surveys of Physics in Biomedicine in Summer of 2012. See Figure 1 for categories.

dimension, the biomedical population had $4.43 \%$ more expert-like responses and $4.04 \%$ fewer novice-like responses on the entrance survey than students exiting Spring term. However, while it had $1.8 \%$ more expert-like responses than the population entering the Fall term algebra-based physics, it also had $1.1 \%$ more novice-like responses.

Overall, the population entering Physics in Biomedicine is more similar to the population entering the subsequent Fall term of algebra-based physics than to the population exiting the preceding Spring term of algebra-based physics.

However, Physics in Biomedicine is consistently outstanding in a few categories. For Personal Interest, students entering Physics in Biomedicine had 14\% more expert-like responses than students leaving Spring term and 16\% more than students entering Fall term. For Real-World Connection, Physics in Biomedicine students came in with $10 \%$ more expert-like responses than Fall term and 4\% more than students leaving Spring term. Superior performance in these categories is expected and persists through the end of Physics in Biomedicine. As one may expect, students electing to take an intensive summer course in physics have an interest in physics and see connections with their world.

Using a two-tailed Z-test, we found that the Overall results were not statistically different $(p=0.61)$, whereas the self-selection results in the Personal Interest category were all significant $(p<0.05)$, with the exception of the comparison of the novice-like responses with students entering Fall term $(p=0.38)$. The Real-World Connection category had $p$ values larger than 0.05 , one exception being the comparison of expert-like responses with students entering Fall term $(p<$ $0.05)$.
The data show that students who elect to take a biomedical physics course see more real-world connections and have more personal interest in physics concepts. Furthermore, preliminary results show that such students may also have a more expert-like attitude overall toward physics than the general population of students beginning or ending the required algebra-based introductory physics sequence.

\section{Attitude Shifts: Comparing Algebra-Based Physics and Physics in Biomedicine}

Here we compare CLASS score shifts among students enrolled in the Physics of Biomedicine course and students in the first term of algebra-based introductory physics. This allows us to make inferences about how the curriculum and instruction affect student attitudes. The first term (Fall) of algebra-based introductory physics covers kinematics, mechanics, and vector algebra and does not encompass medical applications. The physics most relevant to Physics in Biomedicine, light and atomic physics, is not covered until the Spring term of PSU's trimester sequence.

The main purpose of the CLASS is to show how attitudes evolve over time, and the data show that, for these two populations, the Physics in Biomedicine course posted an insignificant negative shift of $-1.2 \%$ (Overall) and the Fall term of algebra-based physics posted a significant negative shift of $-4.2 \%$. We should note that, despite the brief time period during which the Physics of Biomedicine course occurred (3 wk), it was possible to observe shifts in student attitudes (Brown and Sayre 2012). Figures 3 and 4 show the shifts in each category. These are comparable to shifts ranging 
Shifts in Novice-like Responses: Fall Term of Algebra-based Physics and Physics in Biomedicine

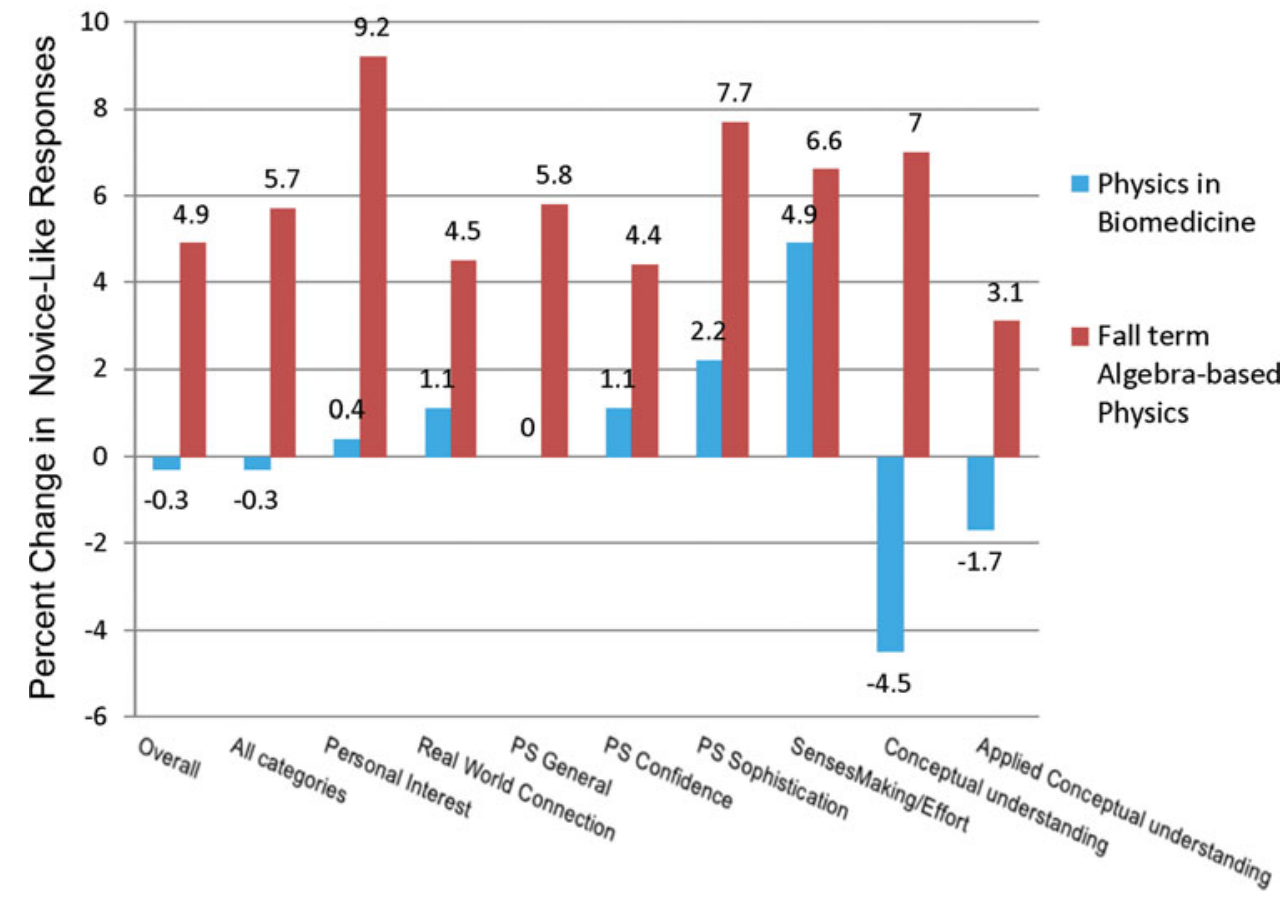

Figure 4. Comparisons of the change in novice-like responses to CLASS survey. Compared are: entrance and exit surveys of introductory algebra-based physics in Fall of 2012 and entrance and exit surveys of Physics in Biomedicine in Summer of 2012. The difference in the shift percentages is the change toward a response of neutral/conflicted. It is necessary to compare these shifts with the shifts in expert-like responses to gauge whether responses were changing from expert to novice or from "neutral/conflicted" to novice or to expert. See Figure 1 for categories.

from -9.8 to $+1.5 \%$ seen in introductory physics courses that include reform and traditional classrooms and calculus- and algebra-based physics (Otero and Gray, 2008).

The category with the greatest improvement was Conceptual Understanding, with a $4.6 \%$ increase in expert-like scores; a $4.5 \%$ reduction in novice-like scores was also posted in that category. A related category, Applied Conceptual Understanding, also saw a decrease in expert-like scores that was less favorable than for algebra-based physics. However, this was a case of responses moving toward neutral, as there was no corresponding increase in novice-like responses. In fact, there was also a decrease in novice-like responses. Conceptual Understanding had statements such as: "I do not expect physics equations to help my understanding of the ideas; they are just for doing calculations" and "Knowledge in physics consists of many disconnected topics."

Two categories suffered large shifts away from expertlike responses and toward novice-like responses for Physics in Biomedicine: Sense-Making/Effort and Problem-Solving Sophistication. Problem-Solving Sophistication differentiates itself from the other problem-solving categories with such statements as: "If I want to apply a method used for solving one physics problem to another problem, the problems must involve very similar situations" and "After I study a topic in physics and feel that I understand it, I have difficulty solving problems on the same topic." Questions in the category of Sense-Making/Effort are: "In doing a physics problem, if my calculation gives a result very different from what I'd expect,
I'd trust the calculation rather than going back through the problem" and "When I solve a physics problem, I explicitly think about which physics ideas apply to the problem."

These shifts appear to be more negative than student shifts in the algebra-based course. It is unclear, at this time, what the causes of these shifts are, but it is an area of interest. The inclusion of sense-making questions within a pedagogical activity has been shown to improve student understanding in physics (Hammer, 2000). An example of such questions is asking students to predict the outcomes of several procedures before starting the experiment and then determining whether the outcome matched their expectation. We speculate that the mathematical models in the curriculum were insufficiently elucidated, especially in the MRI module, which is the last module before the exit survey.

An interesting external comparison can be found in a recent paper by Meredith and Bolker (2012) that has a similar biology-focused reform in algebra-based introductory physics. Meredith and Bolker describe an introductory physics course team-taught by a physicist and a biologist that is extensively modified to be more relevant to life sciences students. They published an Overall shift of $-3.3 \%$ and specified their best category was Real-World Connections, which saw an insignificant shift of $-1.3 \%$.

Although undesired, the negative shift observed in this first assessment of the Physics in Biomedicine course is at the low end of published CLASS data, and more favorable than the shift produced by the algebra-based curriculum and the 
curriculum discussed by Meredith and Bolker. The incoming population to the Physics in Biomedicine course proved to have more personal interest in physics and to be accustomed to seeing more real-world connections in physics. Given the characteristics of this incoming population and the motivations behind the new curriculum the results fail at the ultimate goal of producing a positive shift but meet the expectation of progressing from traditional education.

Because there is not such a stark contrast between the incoming algebra-based physics population and the incoming Physics in Biomedicine population, the benefits brought by further development of the curriculum may be transferable to general physics classrooms. Furthermore, the Physics in Biomedicine curriculum is not consistently weak in areas of strength for the standard curriculum when both expert-like and novice-like responses to the same category are considered (it may be weak in one but not the other). Yet its strong points are consistently strong when looking at both response areas.

The results of this first assessment are promising when considering other results from the local population and similar classes at other universities. The survey also indicates areas for improvement. However, it appears that the biomedical physics course has the desired effect on the students' outlook.

\section{Whole-Course Content Results}

We identified conceptual questions from the PER literature that assessed the physics concepts covered in the Physics in Biomedicine course. Many of the concepts that we tested using the questions from the LSCI (given pre- and postinstruction for the course in its entirety) were taught in the algebra-based sequence. Therefore, most students entering the Physics in Biomedicine course should have been previously exposed to them.

The LSCI as a whole is designed for a course in astrophysics, and most of the questions were not appropriate for our course. R.W. identified questions 5, 7, 8, 10, 13, and 23 as probing content that is particularly relevant for our course. ${ }^{3}$ The data come from an analysis of the LSCI that collected data from more than 4000 students in 69 classes from 31 colleges and universities (Schlingman et al., 2012). In contrast with our students, most of the students in the LSCI study had little experience or prior instruction in physics.

It is not surprising; given the self-selected nature of our sample (students choosing to enroll in another physics course) that we found our students performed better than the astrophysics students on all six questions. To provide a clear comparison of the two populations, we calculated the normalized learning gains for each question (Hake, 1998). The utility of a normalized learning gain is to allow for a comparison of learning across different populations. It is calculated by determining the ratio of percentage of raw gain versus the percentage of maximum possible gain.

$$
g=(\langle\text { post } \%\rangle-\langle\text { pre } \%\rangle) /(\langle 100 \%\rangle-\langle\text { pre } \%\rangle)
$$

${ }^{3}$ LSCI questions should not be widely published in order to protect the integrity of the instrument. To see the LSCI instrument, go to http://ftp.aip.org/epaps/aer/E-AERSCZ-5-2006020/LSCIspring 2006.pdf.
Although we have a small sample of students to consider at this time, the comparisons are informative. These preliminary results indicate that instruction toward these aspects of the Physics of Biomedicine course warrants improvement and revision, because the normalized gains are below those of the astronomy students on questions $5,7,8$, and slightly below for question 23 (see Figure 5). Physics of Biomedicine students appear to do better on questions 10 and 13, which concern wavelength and radiation type and how electron shell behavior causes light emission.

The learning goals of specific modules were examined to see which correlate with questions on the whole-course multiple-choice test; it was determined that, for the validated LSCI questions, only the laser module corresponded strongly to questions 5,7 , and 8 . The light absorption module was loosely associated with questions 10,13 , and 23 . Because medical imaging is a focus of the course, and light is an obvious part of physics enabling such technologies, it was expected that a general improvement in these areas would be seen, and that was the case, as scores improved on all questions. However, it seems that continued curricular improvement and focus is necessary to outperform the astronomy courses.

\section{Individual Unit Example: Light/Absorption}

Learning goals for the individual activities were identified before the start of the Summer quarter. We present here a sample from the Light and Absorption of Light unit, and discuss preliminary outcomes of our assessments. In addition to basic ideas about characteristic wavelengths of light, we probed what students understood about absorption by human tissue.

The worksheet contained a graph that shows the absorption spectra of hemoglobin, oxyhemoglobin, and water, all of which are important to biological imaging and laser surgery. Students had to answer a number of questions on the intensity of different wavelengths of light after penetration into different substances. In the worksheet, students were prompted to relate this to properties of blood and water and were asked how this relates to the use of light in optical microscopy or laser surgery.

The learning goals associated with this section helped to guide the creation of the assessment materials and strategy. The goals are specific to the functioning of lasers but relate to the broader concept goals of the whole course. For example, one goal, stated as "Intensity of light transmitted through tissue drops off with respect to penetration depth," relates to the mathematical and graph reading competencies we wish to instill. Two other goals dealt with the details of how bands of wavelength are attenuated differently in biological tissue. Bands with low attenuation are thought of as "medical imaging windows," and laser surgery requires a careful choice of wavelength that is dependent on the absorption depths for the targeted tissues.

Results from three of the assessment questions in this module are presented here. The first addresses factual knowledge, the second is mathematical in nature, and the third requires applying physics understanding in a medical context. This question required a higher level of understanding and was the most challenging for students. 


\section{Comparison of Instruction Impact}

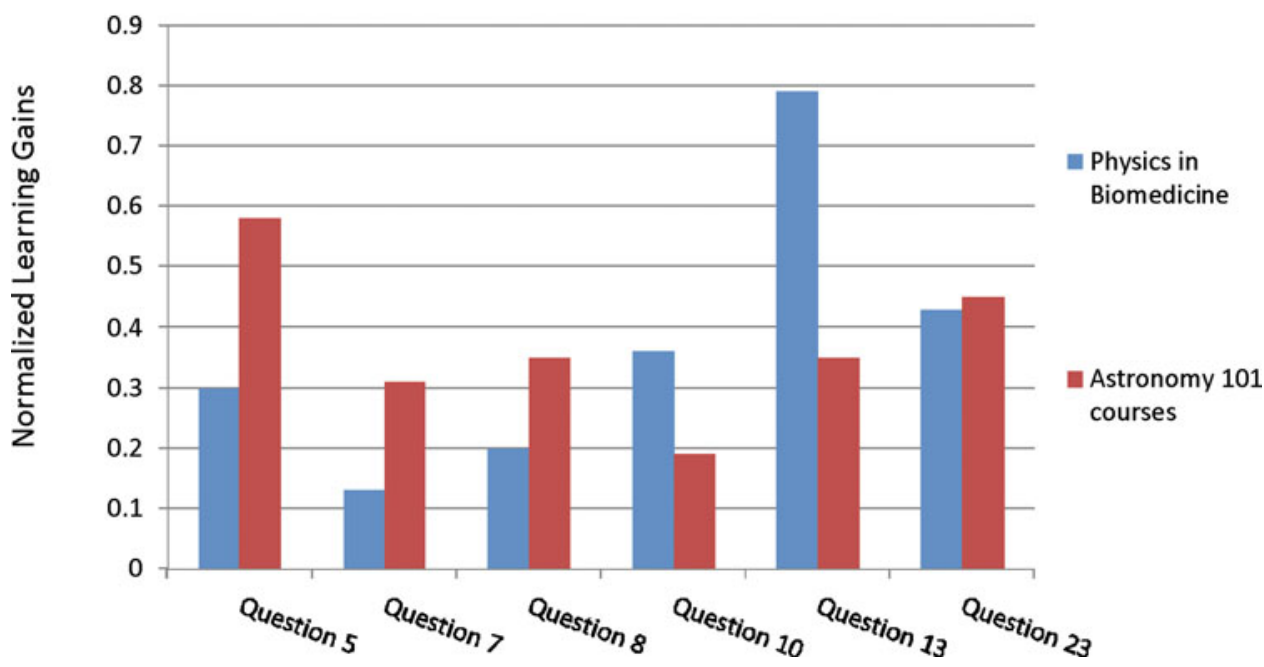

Figure 5. Comparisons of normalized learning gains by Physics in Biomedicine students and introductory astronomy students on choice questions taken from the LSCI. Question 5 asked students to identify which color of light carries the most energy. Questions 7 and 8 asked students to identify movement of an electron between valence shells that corresponds to absorption and emission, respectively. Question 10 asked students to identify which commonplace form of electromagnetic radiation had the shortest wavelength. Question 13 asked students what electron behavior occurs when light is released from an atom. Question 23 asked students to select a true statement from a list comparing wavelength, energy level, and speed of radio waves and visible light. Average astrophysics score in order of appearance on the graph (pre/post): (36/73), (20/45), (14/44), (25/39),(26/52), (14/53); average Physics in Biomedicine scores in the same order (pre/post): (63/74), (50/57), (46/57), (46/65), (80/96), (54/74).

\section{Order of Magnitude of Wavelengths of Visible Light}

Students were asked to identify the characteristic wavelengths of visible light and were offered unit choices of millimeters, micrometers, nanometers, and femtometers. Nearly all of the 23 students (91\%) answered this question correctly before any instruction in the course. After instruction, only one student gave an incorrect response. This concept about characteristic scaling lengths is important, and one that the students seem to understand quite well.

\section{Absorbance versus Penetration Depth}

Light is absorbed by tissue, and as a result, the intensity of light decreases exponentially with respect to penetration depth. We asked students about the functional dependence of this decrease before and after instruction and gave them multiple-choice options of linear, quadratic, logarithmic, and exponential equations. Before instruction, a little more than half $(56 \%)$ of the students gave a correct response to this question. Linear (26\%) and logarithmic (13\%) relationships were the most common incorrect answers. After instruction, nearly all students gave a correct answer (87\%). Students in our population do possess substantial mathematical preparationnearly $40 \%$ have taken calculus 2 and 3-so demonstration of this mathematical understanding is not surprising.

\section{Operations with Light (Cornea vs. Retina)}

A key concept for this module (and the course as a whole) is the understanding of how light interacts with human tissue. Laser surgery relies on specific wavelengths of light being used to interact with tissue. It damages the tissue in a con- trolled manner (e.g., when the cornea is reshaped during LASIK surgery). We probed students' understanding of this idea by asking them the following question: "Would visible light be best used to operate on the cornea or the retina? Why?"

Before instruction, students struggled with this idea. A third of students answered, correctly, that visible light would be best used for operating on the retina. Of those eight students, only half gave a partially correct explanation, describing the cornea as being transparent to visible light without describing any interaction between the retina and visible light.

Students presented a lot of ideas that were not necessarily incorrect, but not really relevant for the question. For example, two students mentioned that it would be used on the cornea, because light does not interact with the cornea. Several noted the brightness of the light needed to be taken into consideration, because it would cause the pupil to contract. There was not a clear identifiable response that could be identified as a student difficulty.

After instruction, 19 of 23 (83\%) students answered correctly, with 15 of them identifying that either the retina does absorb visible light or that the cornea does not. Two of the remaining students who gave correct answers cited that water in the eye does not absorb visible light, which is not wrong but does not provide a complete explanation for using visible light.

In summary, the unit on light and absorption is designed as an introduction to many of the types of interactions students see in the Physics of Biomedicine course, namely, between light and tissue. For the questions we posed, students displayed a high proportion of correct responses after instruction (and in one case before instruction). Although we cannot 
pinpoint the exact activity that caused the improvement, we claim that some part(s) contributed to the shift in correct responses.

\section{Individual Unit Example: Pulse Oximeter}

In this unit, students further investigated the properties of light absorption in human tissue by studying the pulse oximeter, a device that indirectly measures blood oxygen levels by exploiting the different absorption characteristics of hemoglobin and oxyhemoglobin. This activity featured a guest speaker, a short introductory lecture, and a laboratory activity in which students explored the operating principle behind pulse oximetry using bromothymol blue (a $\mathrm{pH}$ indicator) as an analogue for blood. Students also had the opportunity to scrutinize a real pulse oximeter and use it on themselves.

As with the other modules, learning goals were established to guide the instruction and creation of questions on the preand postquizzes. The learning goals were specific to the functioning of the pulse oximeter, but were also relevant to the broader course goals we are assessing. Goals for this section included identifying the key functions of the pulse oximeter: "The pulse-oximeter measures varying oxygen content in the blood and can also measure the patient's pulse." Others relate more strongly to an understanding of the interactions of light and matter, such as: "Students will explain why infrared and red light is used to determine the oxygen levels in blood." Remaining goals addressed the color change of blood, spectral restrictions, and an understanding of how the bromothymol blue experiment relates to operating principles of pulse oximetry. Student responses often focused narrowly on certain aspects of a question or included information relevant to concepts probed on different questions.

We therefore consider the first three questions together and look at the responses as a continuum. The assessment for this module was complicated by the fact that the prequiz was given after the students heard a guest speaker discuss a number of the details of pulse oximetry. Nonetheless, student responses were observed to improve from the prequiz to the postquiz, and a number of students gave a response of "I don't know" to prequiz questions, so it is clear that the content of these ideas was not perfectly conveyed by the lecture. Furthermore, a student was absent on the day of the postquiz, so there are 22 rather than 23 students included in the analysis of the Pulse Oximetry module.

\section{Overall Understanding: Comprehensive Analysis of Questions 1-3}

In the comprehensive analysis of student responses, we looked at three main concepts behind the learning goals: 1) Do students understand the importance of specificity of wavelength? 2) Do students understand how the pulse oximeter exploits the absorbance of $\mathrm{Hb}$ and $\mathrm{HbO}_{2}$ ? 3) Do students understand how constant absorbance by nonblood tissues is handled? Most students went from demonstrating no knowledge or unsophisticated knowledge in the prequiz to demonstrating more sophisticated knowledge on the postquiz. After analyzing responses, we identified characteristics of more sophisticated responses and grouped responses according to those demonstrated characteristics.

\section{Spectra Utilized by Pulse Oximeter}

Students mostly limited their discussion of spectra to questions 1 and 3 . The first question asked students to identify key characteristics of a pulse oximeter. The second question asked them to provide explanations grounded in the physics of light absorption. Students exclusively put discussions of spectral restrictions in these two questions, with the exception of a single response on question 2 in the prequiz.

On the prequiz, 14 students recognized that the pulse oximeter uses restricted wavelengths. On the postquiz, 19 students indicated some notion of wavelength restriction. This may seem like a modest improvement, but the quality of the explanations was far more sophisticated after instruction. Specifically, the number of students correctly identifying that the pulse oximeter uses infrared (IR) and red light increased from two to 13 students. An additional three out of those 19 responses improved without demonstrating a high level of sophistication at the end. On the postquiz, one of these three students demonstrated a vague notion of restricted spectra, and the other two reported that the pulse oximeter uses precisely two wavelengths (without naming them). The remaining three out of 19 students gave the same answer pre- and postinstruction.

\section{Exploitation of Absorbance by Pulse Oximeters}

As before, student ideas were probed across numerous questions, but evidence of their understanding of absorbance was primarily found in question 2 . This question asked students to explain why basic pulse oximeters are inaccurate for anemic patients. It requires a modest level of integrated understanding of the physical meaning of the absorbance measurements used by the pulse oximeter.

Answers varied widely in level of detail and justification, and we note the prevalence of the most commonly presented ideas. On the prequiz, 14 of 22 students demonstrated some level of understanding that pulse oximetry exploits the absorption of light by blood or hemoglobin. Of those, $10 \mathrm{stu}-$ dents vaguely stated that the pulse oximeter uses absorbance characteristics of blood. Only three students made the connection that there is a difference in absorbance for blood (or hemoglobin) between its oxygenated and deoxygenated states. One student made the connection that differences in absorbance of one wavelength lead to a ratio between the $\mathrm{HbO}_{2} / \mathrm{Hb}$ (the most advanced characteristic identified).

After instruction, the number of students demonstrating the minimal understanding of the connection between pulse oximeter and absorption of light by blood or hemoglobin appears unchanged. The number of correct responses only increased from 14 to 15; however, there was an increase in the number of students demonstrating more sophisticated responses. Seven students made the connection between light absorbance and the ratio of the $\mathrm{HbO}_{2} / \mathrm{Hb}$ (up from three before instruction). Of those seven, four students also reported the use of the IR and red light used (compared with zero on the prequiz). Six students recognized that there is a difference in absorption for blood in its states of oxygenation. Two students simply mentioned the less-sophisticated connection that the pulse oximeter exploits absorbance by blood without mentioning particulars. Of note, the student who demonstrated the most complete understanding on the prequizcorrectly identifying that absorbance yields a ratio of $\mathrm{HbO}_{2}$ 
and $\mathrm{Hb}-$ made no reference to any details about absorbance on the postquiz. In no way does this delegitimize our results of general learning gains, but it does indicate that a larger sample size and further assessment is needed before we can get an accurate picture of response sophistication.

\section{The Use of IR and the Role of Nonblood Tissues}

The pulse oximeter is able to function despite the absorbance of nonblood tissues, because it uses IR light (which is absorbed by all tissues equally) as a baseline. The comparison is simple and requires little computing power. (A simple ratio of red/IR absorbances cancels the effect of nonblood tissues and leaves a ratio of absorbance of $\mathrm{HbO}_{2} /\left(\mathrm{Hb}+\mathrm{HbO}_{2}\right)$, which is the oxygen saturation of hemoglobin.) This is another component of a possible answer to question 3. Question 3 asked what makes pulse oximetry inexpensive and convenient and then asked students to elucidate physical properties. The only place absorbance by nonblood tissues was mentioned was in question 3. Three students mentioned it in the prequiz, and a different three students mentioned it in the postquiz. Only one response connected IR to nonblood tissues (postquiz); all of the other responses simply acknowledged absorbance by nonblood tissues, often as added information and without clear integration with the rest of the answer.

Explaining the use of IR in pulse oximetry does require detailed description of precisely which tissue is absorbing which light and how the equations work. Most answers to question 3 had rough descriptions of absorption. Those who mentioned IR never showed whether they understood how its use enabled the pulse oximeter to remove information from nonblood tissues. While the use of IR light and the role of nonblood tissues are important to pulse oximetry, the quiz may not have asked about this clearly enough. Alternatively, the students may simply not value a mathematical description as an explanatory factor.

The Pulse Oximeter unit represents a direct application of physics principles (including those from the Light and Absorption unit) to the use of a medical instrument. In this unit, the number of students' correct answers to our questions did not increase dramatically, but the sophistication of student reasoning improved on several questions. Therefore, we conclude that the instruction in the course did positively impact students understanding as measured by these questions. However, it is uncertain just how acutely (or deeply) we are probing their understanding. Further study is required to ascertain to what extent these questions are probing students' conceptual understanding and to what extent they are merely answering them by parroting memorized facts. Also unclear is the extent to which students could be taught this same information through traditional instructional methods. Although we see an increase in students' correct answers on certain content questions and improved student explanations, further investigation is warranted to determine the breadth and significance of the learning that is occurring in the Physics of Biomedicine course.

\section{CONCLUSIONS}

As the devices used in biology and medicine become more common and advanced, it is increasingly important that students are given the foundations to understand the underlying principles on which those devices are based.

As an elective, the Physics in Biomedicine course attracts mathematically inclined students with favorable attitudes toward physics. As a result of taking the course, the students, overall, do not experience a significant negative shift toward physics or physics learning (whereas most physics instruction leads to a significant negative shift). The course was particularly effective in improving attitudes concerning content understanding, but students experienced a negative shift in areas of sense-making.

Our initial content assessments demonstrate that our students came into the course with some of the relevant physics knowledge, presumably learned from their introductory courses. For relevant questions on the LSCI, our students had uneven results when their normalized learning gains were compared with those of students in a nonmajors course. Individual content assessments showed a mixture of increased correct answers (some gains were small) and more sophisticated reasoning patterns, but additional study is warranted to determine the validity and reliability of our content questions.

\section{FUTURE WORK}

The curricular materials for the Physics of Biomedicine course have undergone the first stage of critical assessment. The next stage of research will be to improve our assessment items and attempt to validate them through the use of one-on-one interviews. We will revise the laboratory activities that include questions meant to push students into sense-making activities. A long-term goal is to adopt these materials for use in the introductory algebra-based physics sequence, in which it has the potential to positively impact a large number of students.

\section{ACKNOWLEDGMENTS}

This work was supported by a grant (TUES 1141078) from the National Science Foundation.

\section{REFERENCES}

Adams WK, Perkins KK, Podolefsky NS, Dubson M, Finkelstein ND, Wieman CE (2006). New instrument for measuring student beliefs about physics and learning physics: The Colorado Learning Attitudes about Science Survey. Phys Rev ST Phys Educ Res 2, 010101.

Ambrose BS, Heron PR, Vokos S, McDermott LC (1999). Student understanding of light as an electromagnetic wave: relating the formalism to physical phenomena. Am J Phys 67, 891-898.

Berenson A, Abelson R (2008). Weighing the costs of the CT scan's look inside the heart. New York Times. www.nytimes.com/ 2008/06/29/business/29scan.html.

Brewe E, Kramer L, O'Brien G (2009). Modeling instruction: positive attitudinal shifts in introductory physics measured with CLASS. Phys Rev ST Phys Educ Res 5, 013102.

Brown T, Sayre EC (2012). Changes in students' epistemologies. Poster presented at the Physics Education Research Conference, held August 1-2, 2012, in Philadelphia, PA.

Elby A (2001). Helping physics students learn how to learn. Am J Phys 69, S54-64. 
Elby A (2012). Epistemological Beliefs Assessment for Physical Science. www2.physics.umd.edu/ elby/EBAPS/home.htm (accessed 31 December 2012).

Hake RR (1998). Interactive-engagement versus traditional methods: a six-thousand-student survey of mechanics test data for introductory physics courses. Am J Phys 66, 64-74.

Halloun IA (1996). Views about Science and Physics Achievement: the VASS story. In: The Changing Role of Physics Departments in Modern Universities: Proceedings of the ICUPE, ed. JR Resish, New York: AIP, 605-613.

Hammer D (2000). Student resources for learning introductory physics. Am J Phys 68, S52-S59.

Hammer D, Redish EF (2009). Reinventing college physics for biologists: explicating an epistemological curriculum. Am J Phys 77, 629-642.

Laws PW, Rosborough PJ, Poodry FJ (1999). Women's responses to an activity-based introductory physics program. Am J Phys 67, S32S37.

McKagan SB, Perkins KK, Wieman CE (2007). Reforming a large lecture modern physics course for engineering majors using a PERbased design. AIP Conf Proc 883, 34-37.

Meredith DC, Bolker JA (2012). Rounding off the cow: challenges and successes in an interdisciplinary physics course for life science students. Am J Phys 80, 913-922.

Moore RW, Foy RLH (1997). The scientific attitude inventory: a revision (SAI II). J Res Sci Teach 34, 327-336.

Mylott EE, Klepteka RJ, Dunlap JC, Widenhorn R (2011). An easily computed laboratory exercise in computed tomography. Eur J Phys $32,227-235$.
Otero VK, Gray KE (2008). Attitudinal gains across multiple universities using the Physics and Everyday Thinking curriculum. Phys Rev ST Phys Educ Res 4, 020104.

Perkins K, Adams W, Dubson M, Finkelstein N, Reid S, Wieman C, LeMaster R (2006). PhET: interactive simulations for teaching and learning physics. Phys Teach 44, 18.

Redish EF, Hammer D (2009). Reinventing college physics for biologists: explicating an epistemological curriculum. Am J Phys 77, 629-642.

Redish EF, Steinberg RN, Saul JM (1998). Student expectations in introductory physics. Am J Phys 66, 212-224.

Schlingman WM, Prather EE, Wallace CS, Rudolph AL, Brissenden G (2012). A classical test theory analysis of the Light and Spectroscopy Concept Inventory national study data set. Astron Educ Rev 11, 010107.

University of Colorado at Boulder (2011). PhET: Online Simulations. http:/ / phet.colorado.edu (accessed 31 December 2012).

Vokos S, Shaffer PS, Ambrose BS, McDermott LC (2000). Student understanding of the wave nature of matter: diffraction and interference of particles. Am J Phys 68, S42-51.

Wald ML (2010). Cancer risks debated for type of x-ray scan. New York Times. www.nytimes.com/2010/01/09/health/ 09scanner.html (accessed 31 December 2012).

Watkins J, Coffey JE, Redish EF, Cooke TJ (2012). Disciplinary authenticity: enriching the reforms of introductory physics courses for life-science students. Phys Rev ST Phys Educ Res 8, 010112.

Wiggins Grant P, McTighe J (2005). Understanding by Design, 2nd ed., Upper Saddle River, NJ: Prentice Hall, 370. 